\title{
EL TRATAMIENTO DE LOS PROBLEMAS DE LENGUAJE EN LA PARÁLISIS CEREBRAL INFANTIL*
}

\author{
Por Miguel Puyuelo Sanclemente \\ Logopeda. Unidad de Desarrollo Psicomotor. Hospital San Juan de Dios. Barcelona
}

\section{M. ${ }^{a}$ Victoria Sanz González}

Ayudante del Departamento de Foniatría y Logopedia. Hospital San Juan de Dios. Zaragoza

\section{INTRODUCCIÓN}

$\mathrm{I}$ NTENTAR resumir en unas hojas, aunque fueran muchas, todo lo que corresponde a este tratamiento es una labor compleja y arriesgada pues, al intentar resumir una experiencia clínica y dar unas pautas metodológicas respecto al modo como debe realizarse, corremos el riesgo de dar a entender que con seguir unas directrices ya está todo hecho. La realidad cotidiana nos demuestra que, aunque todo tratamiento debe basarse en una metodología rigurosa, ella no debe eliminar la flexibilidad del terapeuta, cuya principal habilidad quizá radica en saberse adaptar a las diferentes realidades de cada paciente. A partir de ahí, y con una colaboración activa e interesada de éste se inicia la rehabilitación. Quienes trabajamos en ella sabemos que durante bastantes días el paciente nos ofrecerá una buena colaboración y rendimiento; pero habrá otros en que vendrá cansado o disgustado del colegio..., o nos traerá un juguete que le fascina; o estará preocupado porque no puede jugar a fútbol; o nos querrá contar que la víspera estuvo jugando en casa con su novia del cole... Otras veces serán los padres quienes nos requerirán hasta el punto que en más de una ocasión la sesión será sobre todo de apoyo a éstos. Todo esto responde a situaciones reales y frecuentes a partir de las cuales debemos empezar la rehabilitación: es el

* Nuestro agradecimiento a Michel Le Metayer con quien seguimos un curso de perfeccionamiento en Logopedia en el Hospital de Bicêtre, en París, así como al profesor Guy Thardieu que supo inculcarnos su afán de investigación. Igualmente debemos agradecer a Agnès Wenstein, de Suiza, profesora de Logopedia en los cursos de formación Bobath, sus útiles enseñanzas. mismo paciente quien nos marca, al principio, el camino.

Por ello la rehabilitación de la Parálisis Cerebral presenta numerosas dificultades de ejecución relacionadas además con la afectación motriz. Hay toda una serie de técnicas que el logopeda debe saber emplear.

\section{PROBLEMAS DE LENGUAJE EN LA PARÁLISIS CEREBRAL ${ }^{1}$}

Comunicación:

- Lenguaje:

- receptivo,

- expresivo.

- Voz:

- respiración,

- fonación,

- emisión vocal,

- resonancia.

- Articulación fonética:

Aspectos implicados en la fuerza, precisión y coordinación de los movimientos de la boca, así como en la correcta percepción de los diferentes fonemas y de las oposiciones fonológicas.

Sabemos que el problema principal de la P.C. con respecto al lenguaje expresivo es el acto motor del habla y es aquí donde más centramos el trabajo de

1. Para mayor ampliación véase: M. Puyuelo Sanclemente: «Logopedia y Parálisis Cerebral Infantil». Rev. Logop. Fonoaud., 1982, Vol. I, n. ${ }^{o} 4$, pp. 211-220. 
rehabilitación. Pero debemos tener en cuenta que, además, la adquisición normal del lenguaje puede estar alterada. Siguiendo la clasificación hecha al principio veamos el primer aspecto:

\section{Comunicación}

Sabemos que, durante el primer año, el contacto y el seguimiento ocular, las sensaciones táctiles, el tono muscular, los gestos..., a través del intercambio que el niño tiene con la madre y con los familiares más próximos, es definitivo para el establecimiento de un deseo y una capacidad de comunicación. En el niño con P.C., y debido a su problema motor general, estas experiencias pueden ser más reducidas que en un niño normal de donde queda mermada la capacidad de emisión. Más adelante, la voluntad de comunicación, puede quedar también notablemente reducida debido a la dificultad de emisión; en ocasiones llegan a inhibirse al tener dificultades para hablar y para hacerse entender. Portmann (1982) describe, al principio de su cuadro referido a «comunicación»:

\section{Idea.}

Formación de lenguaje interior.

Voluntad de emisión.

\section{Córtex motor.}

En muchos pacientes con P.C., aun cuando haya una lesión cortical, la voluntad de emisión ya se encuentra inhibida debido a sus dificultades para hacerse entender.

Igualmente si vemos en el «Modelo de situación de comunicación lingüística» descrito por Rondal (1980) cuando al principio dice:

\section{Locutor}

\section{Motivación}

\section{Dominio de la situación}

Representación interna de la experiencia previa,

siendo precisamente el primer aspecto referido a motivación y a dominio de la situación lo que más dificultades implica. A la hora del tratamiento, esta falta de deseo por la comunicación, y más específica- mente por la comunicación oral, debe constituir el primer aspecto sobre el que incidir. En efecto, si tenemos a un niño frustrado por lo limitado de sus posibilidades, difícilmente podremos trabajar en él los demás aspectos.

Por otro lado, si pensamos en las funciones del lenguaje infantil como las describe Halliday (1975):

- función instrumental,

- reguladora,

- interactiva,

- personal,

- heurística,

- imaginativa,

- informativa,

vemos que en el P.C. estas funciones pueden estar dificultadas sobre todo las tres últimas, que implican un fuerte intercambio de opinión con el medio.

En cuanto al aspecto de lenguaje receptivo-lenguaje expresivo, debemos tener en cuenta que un número elevado de nuestros pacientes, entre el 40 y el $50 \%$, tendrá algún grado de deficiencia mental $\mathrm{y}$, unido a él, un déficit de lenguaje.

En cuanto a un retraso evolutivo del lenguaje, esto es, que el nivel de lenguaje sea inferior al de inteligencia general, no es frecuente, como dice la doctora Claude-Chevrie (1975): «Los retrasos graves del lenguaje no son frecuentes en el P.C. y están lejos de representar la causa más frecuentes de sus trastornos». «La expresión del lenguaje, la expresión oral, puede estar considerablemente reducida debido a la disartria, pero ello no implica que el lenguaje esté retardado. Se podrá constatar en estos niños que, cuando hayan adquirido un lenguaje escrito a la edad normal, podrán expresarse correctamente. Es difícil determinar, por ejemplo, si la utilización sistemática de la palabra frase es debida a retraso o a problema motor.»

Por todo ello, en este trabajo nos vamos a centrar en la rehabilitación de los otros aspectos citados: voz y articulación.

Ello no significa que no vayamos a emplear numerosos ejercicios de lenguaje asociados a este trabajo. Sin embargo, no nos referiremos al trabajo específico de lenguaje porque, de existir un retraso mental o un retraso evolutivo, el trabajo seguirá las mismas pautas que en un niño sin P.C. 
La comunicación sí debemos tenerla en cuenta desde el principio favoreciendo, sobre todo: el balbuceo (explicado más adelante), las experiencias sociales y educativas normales (guardería, actos de barrio, grupos de excursionistas...) y, como especialistas, procurando que el paciente haga un uso adecuado de sus posibilidades de emisión vocal, que las acepte y, si son muy limitadas, proporcionándole un método alternativo de comunicación.

\section{EL TRATAMIENTO}

\section{Bases del tratamiento}

Sabemos que el habla en un niño normal comienza en un determinado momento del desarrollo psicomotor, y que en ella está implicada la coordinación de numerosos grupos musculares, sensaciones, percepciones... Por ello, y para poder llegar al control y selectividad que requiere el acto motor del habla, es necesario un desarrollo de los patrones generales del movimiento. De esta forma vemos que, una vez desaparecidos los reflejos orales, empieza a comer sólidos; cuando tiene un buen control de cabeza, y más cuando empieza a caminar, es capaz de hacer la pinza con precisión... es entonces cuando empiezan las primeras palabras.

- En cada momento del tratamiento el logopeda ha de saber evaluar el nivel motor del paciente para saber si existe actividad refleja anormal y el estado del tono muscular; para saber si tiene que inhibir algún movimiento, aumentar el tono muscular, relajar... Por ello, antes de empezar la emisión oral debemos cuidar que el tono muscular sea normal. Es importante que a la hora de hablar, el paciente no deba preocuparse por su cuerpo: esto es, que no vaya a aparecer un reflejo tónico-asimétrico, en el momento de comenzar la emisión; o que tenga un tono muscular tan bajo que hable sin apenas mover la boca. Por ello durante cierto tiempo se trabaja con el paciente controlado para, más adelante, dejarle en posiciones más libres: sentado, de pie, andando...

- El tratamiento debe de ser gradual de acuerdo con las posibilidades motrices del paciente. Por ejem- plo, no empezamos realizando ejercicios de dicción con frases o relatos breves en la primera sesión, si el niño no controla el reflejo de morder o presenta un espasmo en extensión cada vez que intenta hablar. Primero trataremos de facilitar los movimientos de lengua y labios hasta que, de forma progresiva, lleguemos a la emisión de palabras breves.

- La colaboración de la familia es fundamental a lo largo de todo este proceso pues muchos de los aspectos del tratamiento deben trabajarse también en casa: alimentación, balbuceo, emisión oral... Además, el hogar constituye el primer sitio en donde debemos intentar la generalización de lo aprendido durante la sesión de rehabilitación: ésta puede estar muy bien llevada, y el niño desarrollar en ella toda una serie de hábitos de emisión vocal y luego... en casa, en el colegio, utilizar los patrones patológicos de emisión vocal.

- Debemos respetar los centros de interés. Tengamos en cuenta que puede ocurrir que el paciente tenga, por ejemplo, una edad cronológica de 6 años cuando nos llega por primera vez y su problema motor sea grave. Aunque sea necesario controlarlo y empezar por ejercicios de emisión vocal sencillos, la dinámica será distinta de la aplicada a un paciente de dos años con la misma afección.

- Debemos ser conscientes de los límites del tratamiento en cada caso y tener en cuenta que no buscamos locutores con una voz perfecta sino que el habla sea comprensible y tenga una cierta fluidez para poderse adaptar a una situación social y cultural.

\section{Aspectos metodológicos del tratamiento}

- Seguir las pautas del desarrollo normal.

- Facilitación del desarrollo neuromuscular.

- Inhibición de los patrones anormales de movimiento, facilitación del movimiento normal.

- Criterios diferenciales que deben tenerse en cuenta:

- el tono muscular: alto,

hipotónico, variable; 
- parte del cuerpo más afectada:

extremidades superiores, extremidades inferiores

lado derecho, lado izquierdo.

- Grado de afección de la parte afectada: grave, medio, ligero.

- Estudio de conjunto de los movimientos de la cabeza, tronco, cadera y extremidades, para valorar cómo influye todo ello en la emisión de la voz.

- Momento más adecuado de empezar el tratamiento.

- Colaboración familiar.

- Objetivos del tratamiento.

- Etapas que deben tenerse en cuenta.

- Nivel intelectual.

- Trastornos asociados.

\section{Apartados del tratamiento}

- Alimentación.

- Facilitación postural.

- Comunicación.

- Estrecha colaboración con la familia.

- Papel importante del juego.

- Praxias bucolinguales.

- Tratamiento del babeo.

- Respiración.

- Emisión vocal.

- Ejercicios de dicción.

- Métodos de comunicación no verbal.

Debemos aclarar que cada apartado, aunque tenga sus objetivos y su programación propia, no se trabaja por separado sino que se integra dentro de la dinámica de la sesión de reeducación. Ésta debe tener una continuidad y ha de tenerse la suficiente habilidad para ir coordinando unos apartados con otros y con tareas originales, dando además una cierta iniciativa al paciente. Muchas veces hablaremos de inhibición del patrón patológico anormal y de favorecer el movimiento normal; aun cuando esto implica un cierto control de la motricidad del paciente por parte del logopeda, no quiere decir que tengamos al paciente en una posición supercontrolada, en la que lo único que podrá hacer será hablar correctamente, sino que le ayudaremos a realizar los movimientos. Y, si en un momento determinado, estos objetivos no son de interés para él, cambiaremos de metodología.

\section{TRATAMIENTO DE LA ALIMENTACIÓN}

Puede situarse dentro de todo el conjunto de aspectos que se pueden trabajar y estimular desde los primeros meses de vida para conseguir un mejor desarrollo del lenguaje oral como son: mímica facial; coordinación y atención visual; balbuceo; reflejos orales; alimentación.

De hecho, el estudio de la alimentación debe iniciarse con el de los reflejos orales pues, como dice Lidia Coriat (1980), coordinados entre sí constituyen una sinergia de acciones reflejas que posibilitan y aseguran la alimentación del recién nacido. Con base en estas estructuras se desarrollará ulteriormente la actividad alimentaria, pero no sólo ella sino las estructuras biológicas que sirven de sustrato anatómico de los reflejos orales y que servirán de soporte para las funciones de coordinación de la boca con las manos y para el uso de los órganos fonoarticulatorios. Por ejemplo: a nadie le escapa la importancia del reflejo de succión que, «coordinado con una deglución correcta, constituyen una secuencia rítmica de movimientos que irán seguidos con el mismo ritmo, y en forma inmediata, por brevísimas detenciones de la respiración».

El no desarrollo de los reflejos orales, o algunas alteraciones en la motricidad de la alimentación, pueden producir una serie de problemas en el P.C. que dificultarán la correcta elocución. Por ejemplo, sabemos que la dirección del crecimiento de los dientes está influida por las presiones de la musculatura labios-mejillas-lengua. De ello se desprende la acción nefasta de la interposición lingual durante la deglución, o de la presión de ésta sobre la cara posterior de los incisivos, que frecuentemente va asociada a malposición lingual en la articulación de algunos fonemas. De todas formas, tengamos en cuenta, como dice Segovia (1977), que «la fisiología de los músculos 
bucofaciales es esencialmente condicionada por el sistema nervioso que determina el tono, la posición de reposo y la posición de los músculos, así como las modalidades de cumplimiento de las diversas funciones de las cuales los músculos orofaciales son los efectores».

Un aspecto que tampoco debemos olvidar es el sentido del gusto. Según Sait-Anne Dargassie y Minkowski, a las 32 semanas de edad gestacional pueden percibirse, en bebés prematuros, gestos de agrado o de rechazo al colocarles en la boca gotas de soluciones dulces o amargas.

\section{Problemas más frecuentes}

- Patrón postural anormal del niño y muchas veces de la madre.

- Movimientos de extensión de nuca y brazos; posturas asimétricas.

- Dificultades de coordinación de la succión, deglución y respiración.

- Deglución con interposición lingual.

- Funcionalidad labial muy reducida, sobre todo en el labio superior.

- Empleo de instrumentos (cuchara, tenedor, vasos especiales, tetinas de biberón alargadas...) no adecuados e incluso perjudiciales. Cucharas grandes y hondas; vasos grandes o vasos educativos con boquilla; baberos en peto, de plástico, que comprimen la faringe del niño; biberones con tetinas largas y excesivamente grandes...

- Incorrecta dinámica de la alimentación por parte de la madre. Por ejemplo: derramar el contenido de la cuchara dentro de la boca, no favoreciendo con ello la funcionalidad de labios, lengua y mejillas. Empleo de cucharas excesivamente grandes y que, además, llegan a tropezar con los dientes.

- A veces la madre, debido a que el niño derrama parte del contenido bucal, emplea cucharas mayores para que así «algo quede dentro de la boca...». Y le da más deprisa... Con todo ello se crea un círculo vicioso en el que el niño, como defensa, sigue derramando la comida y se ve obligado a hacer una protusión lingual constante para no ahogarse.
- Dificultades en la adquisición de la masticación: muerde la cuchara al no disociar los movimientos de los labios y del maxilar inferior.

- Motricidad reducida de la lengua, sólo movimientos laterales y no rotación.

- Frecuente derrame del contenido bucal con diferencias según el tipo de alimento.

\section{Aspectos del tratamiento de alimentación}

Los aspectos relacionados con la alimentación se trabajan desde el primer mes de vida del niño.

Los apartados principales serían:

- posición del niño;

- tipo de instrumentos que utiliza la madre en la comida;

- velocidad con que le da de comer;

- preparación de la zona oral antes de las comidas;

- control bucal, si procede.

Por ejemplo, para dar de comer con la cuchara, los movimientos correctos serían:

- Emplear una cuchara normal que se adapte bien a la boca del niño, poco profunda, más bien plana y que no sea de plástico; ligeramente alargada para que se adapte mejor a la forma de la lengua.

- Cabeza en ligera flexión, procurando que el control de la mandíbula sea bueno.

Evitar los movimientos de extensión, en especial de la nuca y de la boca. Para ello abrir ligeramente la boca y entrar toda la cuchara horizontalmente y en el medio. Luego presionar la lengua con la cuchara, lo que provocará el cierre espontáneo de la boca. Daremos tiempo a que el labio superior sea el que retenga el alimento al retirar la cuchara horizontalmente, sin prisas. Progresivamente intentaremos dejar el control sobre el maxilar.

\section{Las posiciones}

Veamos los diferentes tipos. 


\section{1) Sobre la madre}

Según la edad del niño y el grado de afectación puede ir mejor una u otra (fig. 1).
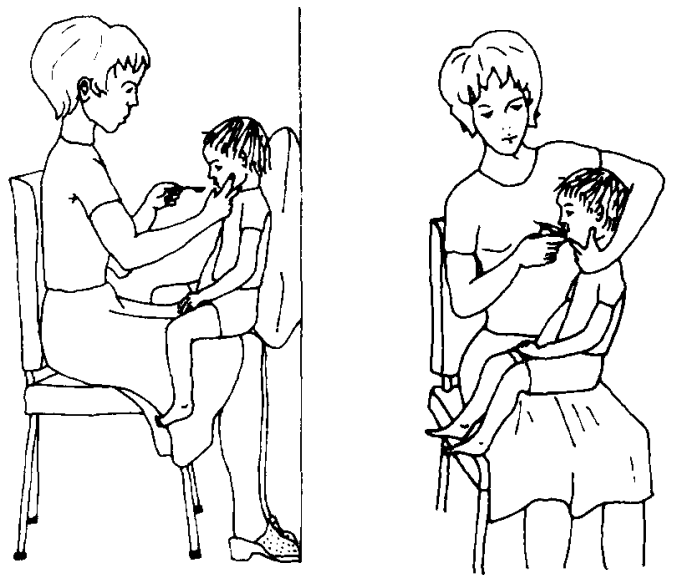

FIG. 1

2) En el plano inclinado (fig. 2).

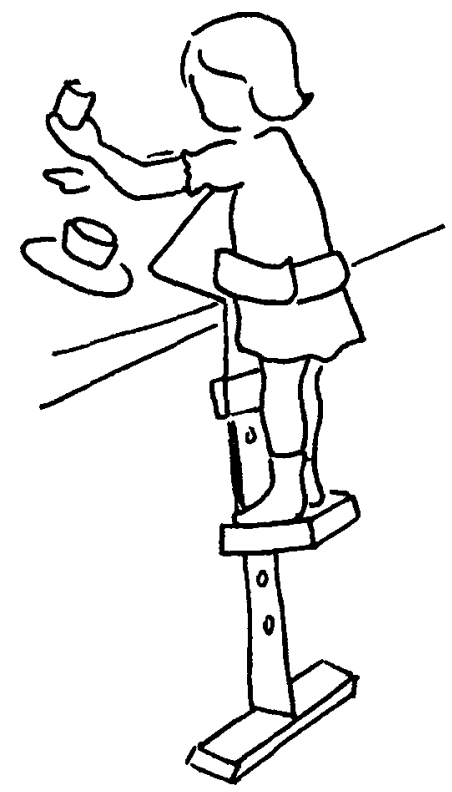

FIG. 2.
3) En una silla triangular (fig. 3).

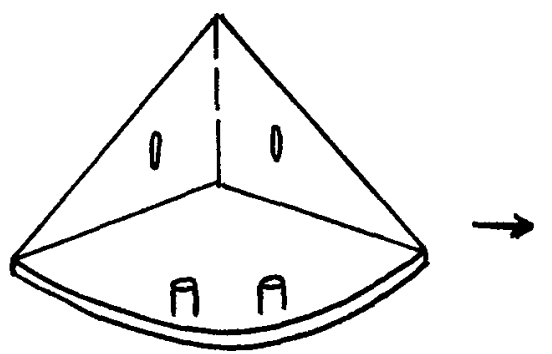

FIG. 3.

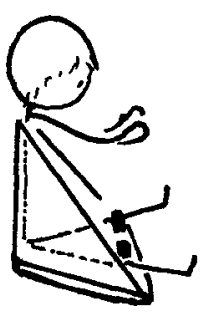

4) En una trona (fig. 4).

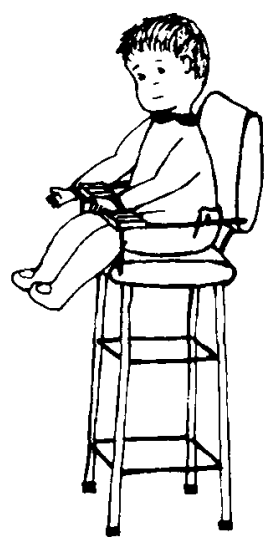

FIG. 4.

5) Sobre un rulo (fig. 5).

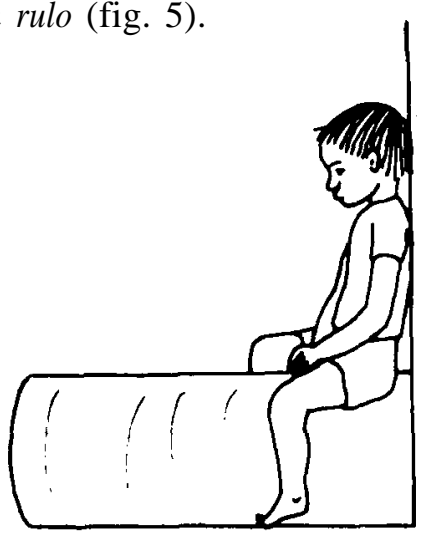

FIG. 5 
6) En una silla normal, pero cuidando de que los apoyos del niño sean correctos, columna, pies...

El empleo de una u otra de las posibilidades estará en función del grado de afectación del niño y de sus necesidades de autonomía. A un niño de 2 o 3 años que está muy afectado, pero intenta y quiere comer solo, no podemos obligarle a permanecer totalmente controlado.

\section{Para beber}

Vaso de plástico que se coloca sobre el labio inferior. Procurar que el líquido caiga regularmente, pero no en cantidad excesiva. Cerrar la boca ejerciendo control para evitar que saque la lengua o haga extensión con la boca.

Vigilar para que no coja el vaso con los dientes. Si es posible dejar el vaso en contacto continuo con el labio inferior, para evitar una sobreestimulación. Procurar que trabajen los labios. Otra posibilidad es que tome el líquido con una pajita flexible, entrenándole primero a que lo haga con la pajita sola. A veces es preciso ayudarle en el movimiento de los labios.

\section{Inicio de la masticación}

Favorecer este apartado es de suma importancia por los progresos que producirá en cuanto a la inhibición de los reflejos orales $\mathrm{y}$, sobre todo, porque implica una serie de movimientos más amplios de la lengua y una gran coordinación a nivel bucolingual.

Si no mastican a la edad adecuada, hay dos factores que deben tenerse en cuenta;

- El grado de afectación matriz a nivel bucolingual. Ello, por ejemplo, puede causar una incapacidad para trasladar el bolo alimenticio con la lengua hacia los molares, o la reducción de la movilidad del maxilar inferior, debida además a una gran espasticidad.

- El grado de motivación para masticar. Por importante que sea el nivel de afectación, siempre se revelan como muy importantes las ganas que el niño tenga de hacerlo. Por ejemplo, puede influir favorablemente el hacerlo en grupo en la guardería; que empiece a hacerlo un hermano algo menor que él; comer con todos en la mesa: jentonces quiere probar la tortilla o la carne de papá!; que empiece masticando alimentos que le gustan; hacerle masticar cuando tiene más apetito, no cuando ya lleva un rato comiendo.

\section{TRATAMIENTO DE LA SENSIBILIDAD DE LA ZONA ORAL Y PERIORAL}

Empezamos con la boca cerrada. Tocamos la cara, sin entrar dentro de la boca. Lo haremos con nuestros dedos, con los suyos o con objetos diversos. Si apoyamos mucho, la estimulación es menor que si hacemos poca presión. Por ello, en los hipersensibles haremos una estimulación fuerte y precisa.

Luego, dentro de la boca: ${ }^{1}$

encías,

paladar duro,

lengua.

\section{Encías}

De delante atrás; haremos tres veces la excitación, sin dejar de apoyar el dedo y esperando a que el niño haya tragado la saliva antes de empezar la estimulación siguiente. El maxilar ha de controlarse con los tres dedos y con la boca ligeramente abierta para que pueda pasar el índice o el meñique.

\section{Paladar duro}

Tres veces de delante atrás, sin ir muy atrás para no provocar la náusea. Cerrar la boca y esperar a que trague.

1. Método desarrollado por K. y B. Bobath y Rood (Londres). 


\section{Lengua}

Controlar el maxilar entreabierto, el dedo sobre la lengua. Si es rígida, hacemos presión y luego movimientos laterales.

Si está hacia atrás primero la cogemos y la llevamos hacia delante y luego la descontraemos.

$\mathrm{Si}$ es hipotónica, se hacen co-contracciones.

Iniciar el tratamiento en supino y mantener la cabeza del paciente siempre en flexión.

\section{FACILITACIÓN DEL BALBUCEO}

Se trata de aprovechar situaciones placenteras. El niño debe permanecer en una posición correcta y con un estímulo agradable (la presencia de alguien de la familia, juguetes que capten su atención...).

Crikmay nos habla de la madre, pero creo que es importante señalar que a veces «los abuelos» tienen un papel sorprendente. He visto a niños con afecciones graves que ante las consignas de uno de los abuelos realizaban algunas manipulaciones que nosotros hubiéramos conseguido sólo después de semanas de motivación. O el caso de una niña cuya primera palabra fue «papá» y la segunda «yaya María». Otro niño, muy afectado, que dice: «Aan-Joan, yaya, aqua-agua apo-guapo, tía, ete-tete», pero no dice papá (el padre no vive con la familia), ni tampoco pipi, caca...

Parece claro que, de cara a la adquisición del primer vocabulario y aunque la ejecución de determinadas palabras implique mayor dificultad, es más probable que el niño comience con nombres de familiares o algún objeto que capte su atención (coche, agua, pan...), antes que con otras palabras fonéticamente más sencillas.

La Unesco (1976), dentro de un volumen dedicado al niño de 0 a 6 años nos habla de lo importante que es estimular el balbuceo:

«Hablarle a menudo, durante la alimentación, aseo...».

«Enseñarle palabras sencillas, repetir las que él dice...».
Ronda1 (1980) nos explica todas las secuencias del intervalo verbal entre madre y niño durante todo el primer año.

En el niño con patología debemos favorecer el contacto ocular y aumentar nuestras emisiones verbales con él.

En otras ocasiones, el nacimiento de un nuevo hermano ha provocado una nueva dinámica intrafamiliar. Así, niños que a los 4 o 5 años no hablaban, pues tenían un problema motor grave, al empezar el nuevo hermano sus primeras palabras, al año, se esforzaron en ampliar su repertorio con logros realmente importantes.

No cabe duda de que a lo largo del día habrá momentos que serán más propicios: éste será el momento que deberá aprovecharse. Se tratará de reforzar todos los sonidos que el niño diga: imitándole y sonriéndole, intentaremos aumentar el número de tales sonidos, las variaciones tonales... para más adelante intentar introducir otros sonidos nuevos.

Por otro lado, no se puede ignorar que el movimiento normal favorece la aparición de estos sonidos. Por ello no nos limitaremos a favorecer el balbuceo en la cama y con el niño inmóvil, sino que intentaremos que voltee, juegue con objetos, los lleve de un lado a otro de la cama, colchoneta o mesa, que esté sentado. Porque todo ello propiciará un tono muscular general más favorable a la emisión de sonido.

\section{EJERCICIOS DE FACILITACIÓN POSTURAL}

El objetivo de estos ejercicios es conseguir una postura lo más parecida posible a la posición normal adecuada, que favorece la emisión vocal del paciente tanto si está sentado o de pie como andando.

\section{Métodos}

- Favorecer el movimiento normal.

- Favorecer el tono muscular normal. 
- Favorecer los movimientos del sistema fonoarticulatorio.

Los ejercicios incluyen: movilizaciones pasivas del paciente; instalación adecuada; ejercicios de rotación y de movilizaciones pasivas en los espásticos para relajarlos; ejercicios para evitar que se fijen en una postura determinada; ejercicios de co-contracción de tapping en los atetósicos para normalizar el tono; ejercicios sobre los músculos de la nuca y el cuello para relajar la cabeza y luego el maxilar inferior.

\section{Criterios diferenciales}

Esta facilitación será diferente según el tipo de lesión del paciente y según su grado:

- En el espástico, la facilitación será dinámica y tendrán gran importancia los ejercicios de rotación.

- En el atetósico, se buscará una posición estable, en la que se produzca el mínimo posible de distonías durante la emisión vocal. Se intentará también aumentar y estabilizar el tono muscular según proceda.

En cuanto a la postura en general, se buscará:

- Que sea simétrica y estable. Pero el paciente ha de poder moverse y colaborar activamente en el mantenimiento postural.

- Se procurará que la posición de la cadera esté en relación con la del tronco.

- El tronco permanecerá recto y con los músculos activos.

- Los hombros mantendrán una postura correcta y no se bloquearán en el momento de la respiración o en la emisión vocal.

- La posición de la nuca será la adecuada, sin favorecer los movimientos de los extensores.

- La boca permanecerá cerrada cuando corresponda.

Por supuesto, en la última fase será el propio paciente quien realizará estos ejercicios y conseguirá integrarlos en su actividad cotidiana.

\section{TRATAMIENTO DE LAS PRAXIAS BUCOLINGUALES}

Ya hemos visto anteriormente que el primer trabajo correspondiente a las praxias de la zona oral se desarrolla por: el tratamiento preparatorio a la alimentación, el tratamiento de la alimentación y favoreciendo el balbuceo.

Dentro de la sesión de tratamiento logopédico, las praxias se trabajan junto con el control motor general antes de empezar el trabajo de articulación. Como paso previo a las praxias, debemos tener en cuenta los ejercicios de facilitación postural y los que influyen en el tono muscular de la zona oral. Por ello intentaremos:

- normalizar el tono muscular general;

- controlar la cabeza, cuello y nuca;

- controlar el maxilar inferior.

A nivel de la cabeza y cuello intentaremos evitar la extensión, las asimetrías y que la cabeza se fije al cuello.

\section{Aspectos del tratamiento}

- Si la cabeza y los músculos faciales presentan hipertonía, intentaremos normalizarlos relajando nuca y mejillas.

- Si la musculatura está fláccida, podemos hacer tapping con las manos y co-contracciones de cabeza.

- También realizaremos tapping sobre la lengua, de detrás adelante, o con co-contracciones.

- Si la lengua está rígida, haremos primero presión y luego movimientos laterales. Si está hacia atrás, la cogeremos llevándola hacia delante y la relajaremos con el dedo llevándola de derecha a izquierda.

- Si la cabeza y el tronco están rígidos en extensión, intentaremos relajarlos con rotaciones suaves.

- Si vemos que el maxilar inferior está hipotónico, controlaremos la boca y a partir de ahí, co-contracciones.

- $\mathrm{Si}$, por el contrario, está muy rígido, lo relajaremos con movimientos circulares realizados desde 
detrás de la cabeza, o por delante, sujetando suavemente el mentón en pinza.

- Labios: si están con un tono alto, los cogeremos entre el dedo índice y medio y realizaremos pequeñas vibraciones hasta relajarlos y conseguir una posición normal.

- Si, por el contrario, hay una base hipotónica, haremos tapping sobre ambos.

- Todos estos ejercicios se realizan en una primera fase del tratamiento. Posteriormente se deberán

\section{TABLA I. - Ejercicios de soplo}

\section{Con velas pequeñas o grandes:}

Soplar hasta apagarlas.

Soplar sin apagarlas, sólo mover la llama.

- Con una pelota de ping-pong y un muñeco de papel:

Soplar la pelota en dirección al muñeco hasta que lo derribemos.

Si el soplo no tiene la suficiente potencia como para apagar una vela o empujar una pelota de ping-pong, hacerle soplar una flauta.

- Figuras de papel o de algodón

Preparar varias figuras de papel o de algodón y colocarlas soplando, en un sitio prefijado de la mesa.

\section{TABLA II. - Ejercicios de lengua}

- Sacar y meter la lengua con los labios cerrados.

- Serie de 5 movimientos en los que se sacará la lengua, abriendo simultáneamente la boca; al meter la lengua, se cerrará la boca. Se descansará durante 10 segundos, que el logopeda contará en voz alta mientras el paciente mantiene la boca cerrada.

- Control postural de la lengua: mantenerla inmóvil durante determinado tiempo y en determinada posición.

Ejemplo: sacarla con los labios cerrados, mantenerla inmóvil durante 5 o 10 segundos y luego volver a la posición inicial.

- Hacerla vibrar entre los labios.

- En ocasiones es conveniente emplear un espejo de pequeñas dimensiones en el que sólo se vean las mejillas y la zona oral.

- Colocar objetos de formas diferentes dentro de la lengua y hacer que los reconozca.

Ejemplo: algo redondo y algo cuadrado; algo rugoso y algo liso; misma forma pero diferente peso.

- Ejercicios circulares dentro de la boca cerrada, como si tuviéramos un caramelo grande.
TABLA III. - Ejercicios de labios

- Oprimirlos fuerte uno contra otro en una posición simétrica. En esta posición, oponer resistencia a que sean abiertos por el dedo del terapeuta.

- Proyectar los labios en una U muy pronunciada; a continuación pasar a una E y luego a reposo.

- Mover los labios como para pronunciar A, O, U, E, I exagerando el movimiento.

- Colocar entre los labios una caña de sorbete; que la aguante; tirar y que el paciente haga fuerza para que no se la quiten (más adelante se realiza con objetos más gruesos).

- Hacer vibrar los labios.

- Con el labio superior tapar el inferior y recíprocamente.

TABLA IV. - Ejercicios de mejillas

- Son de bastante interés para una correcta articulación y dicción. En parte ya se habrán trabajado en ejercicios anteriores, pero veamos algunos a título indicativo:

- Hinchar las dos mejillas a la vez.

- Hinchar alternativamente la derecha y la izquierda.

- En alguna ocasión puede ser útil pintar un lado de un color y el otro de otro, o pegar dibujos diferentes en cada mejilla.

- Que hinche la mejilla pintada, pero el logopeda opone una ligera resistencia con su mano.

- Empujar con la lengua las mejillas en todas las direcciones.

- Hincharlas como si fueran un globo; luego se deshinchan poco a poco.

- Hacer como que masticamos el bocadillo predilecto: lentamente, de prisa, descanso para beber... seguimos.

realizar ejercicios voluntarios de praxias bucolinguales. En las tablas I-IV proponemos algunos ejemplos al respecto.

Precisemos que en estos ejercicios nos interesa de igual manera la consecución del ejercicio como la forma de realizarlo. No se trata de realizar el movimiento de forma rápida; las posiciones intermedias también tienen importancia.

Ejemplo: no se trata de que apague la llama de una vela en extensión de tronco, sino sentado correctamente y con la cabeza en ligera flexión. No se trata de que pueda llevar la lengua fuera de la boca, sino que pueda hacerlo lentamente y deprisa. 


\section{ARTICULACIÓN}

En ocasiones tendremos que ayudarle para que aprenda los puntos correctos de la articulación de algunos sonidos. Debemos pues enseñarle el punto y modo de articulación. Para esto puede sernos útil:

- tener un espejo grande y otro pequeño;

- la posición sedente;

- un guíalenguas;

- enseñarle sobre nosotros mismos;

- luego lo hará él.

\section{Ejercicios orientativos}

La I: Hacer sonreír varias veces al niño. Debe apoyar la punta de la lengua sobre la cara interna de los incisivos inferiores y sonreír emitiendo sonido. Procurar que la separación de labios y dientes no sea excesiva.

La O: Si no coloca los labios en la posición adecuada para facilitar el movimiento, le podemos poner una cartulina enrollada entre los labios.

La U: Si no le sale, podemos introducir un lápiz entre los labios, a la vez que le invitamos a realizar el sonido.

La P: Que sople sobre velas. Luego que lo haga con explosión. Hacérselo notar sobre su propia mano.

La B: Se puede obtener a partir del soplo. Si no, podemos favorecerla colocando nuestro dedo entre sus labios para favorecer la posición. Al colocar el dedo, podemos percibir también las vibraciones de la producción del fonema.

La Z Y la C: Se pueden hacer también a partir del soplo. Que sople primero diferentes objetos sobre la mesa; a continuación, que se muerda suavemente la punta de la lengua y sople sobre unos papeles.

La M: Pronunciar la A y luego cerrar los labios para pronunciar la $\mathrm{M}$.

La N: Le haremos colocar la punta de la lengua contra los incisivos superiores. Le podemos hacer notar las vibraciones que se producen en la nariz cuando la emite.

\section{TRATAMIENTO DEL BABEO}

Antes de iniciar este tratamiento hay que valorar mucho los posibles resultados, pues es un aspecto del tratamiento que siempre crea ansiedad y de resultados muy inciertos. Los apartados que se tienen en cuenta de cara al tratamiento son los siguientes:

- Ejercicios de alimentación.

- Si el paciente mantiene o no la cabeza.

- Trabajo de la respiración nasal. Trabajo de los músculos implicados, por medio de ejercicios de soplo y de sorber.

- Ejercicios de deglución regular.

- Colocación de prótesis dentro de la boca.

- Soluciones quirúrgicas.

En una primera fase se intenta inhibir el babeo actuando sobre los músculos de la zona oral y perioral, normalizando su tono muscular y sus funciones y, además, trabajando la deglución de forma que sea regular y automática. En la mayoría de los casos se consiguen buenos resultados trabajándolo a cortos intervalos cada día y de forma regular. Pero hay casos en los que no se consigue inhibir el babeo, que llega a constituir un auténtico problema higiénico y social (por ejemplo, adolescentes que no lo pueden inhibir). Para tratarlo, diversos autores recurren a prótesis dentro de la cavidad bucal o incluso a operaciones. Por ello el doctor Grenier, de Bayona, habla de un aparato antibabeo (1979): «El aparato antibabeo es una protesis dentaria semimóvil, que crea estimulaciones de movimientos a fin de impedir la formación de una reserva de saliva en la parte anterior de la boca; suprimir la acumulación, si ésta se produce y colocar las mandíbulas, lengua y labios en posición de deglución salival.»

El aparato descrito por Grenier consta de un elemento predentario que se coloca entre el labio y las encías, de forma que provoca movimientos del orbicular. Un segundo elemento retrodentario se coloca detrás de los incisivos y provoca movimientos de la lengua y de las mandíbulas, que se aprietan; con ello se evita que la saliva salga fuera de la boca y se facilita la deglución. Por supuesto, este aparato requiere una adaptación individual y algo prolongada; aunque 
su uso no está muy extendido, en ocasiones se ha revelado muy eficaz.

H. Haberfellner (1980) dice : «En ocasiones la terapia de la zona oral, de K. y B. Bobath y de Rood, no es suficiente; por ello desde hace 6 años hemos empleado algunos aparatos utilizados en ortodoncia clásica. Se trata del monobloc-activador de Andersen y Haupl y el regulador funcional de Frankel.» Estos aparatos corrigen la posición de la lengua, lo que, a su vez, contribuye a normalizar la función del velo palatino y también la de toda la región faríngea. Estos aparatos se utilizan, al principio, durante dos o tres minutos al día, dos o tres veces y, después, únicamente por la noche. Con el uso de estas prótesis se busca mejorar la posición habitual de los labios, el control de los movimientos linguales, las funciones nutritivas y la articulación de las palabras.

H. Haberfellner y Bernt Rossiwall (1977), refiriéndose a estos aparatos y a sus resultados, comentaban: «Los niños con P.C. y con importantes trastornos sensoriales han sido tratados con aparatos funcionales, habiéndose observado un razonable éxito terapéutico en el control del babeo y masticación-deglución, mejorando la disfunción perioral, la disfonía y la disartitulación.»

Desde hace algunos años se intentan aplicar igualmente técnicas quirúrgicas que varían sustancialmente de unos autores a otros. Citaremos dos:

Garry S. Brody en Control of Drooling by Translocation of Parotid Duct and Extirpation of Mandibular Gland (1977) dice: «Control del babeo en el P.C. y en el retrasado mental: la técnica de Wilkie modificada, consiste en una resección de los submaxilares y en un trasplante del canal parótido, se ha mostrado eficaz en más del $90 \%$ del control del babeo en 120 individuos, seguidos durante 1 a 6 años: la intervención es segura, eficaz y parece definitiva.»

H. Grady Arnold y Charles W. Gross en Transtympanic Neurectomy: a Solution to Drooling Problems (1977), no se manifiestan partidarios de la extirpación de las glándulas parotídeas por el riesgo que supone actuar sobre el nervio facial y por las complicaciones estéticas que produce esta técnica. Sin embargo, la técnica por ellos descrita parece más sencilla y sin tantos problemas postoperatorios. Como conclusión de su trabajo comentan: «A treinta individuos con lesiones cerebrales se les ha aplicado una neurectomía trastimpánica por babeo severo. Han sido estudiados postoperatoriamente durante 14 meses y el nivel de mejoría ha sido del $87 \%$. No se han observado complicaciones serias o irreversibles y la calidad de vida de los individuos no ha sido reducida.»

El problema del babeo hay que relacionarlo con unas causas sensoriomotrices evidentes que provocan problemas higiénicos y sociales. Sus posibilidades de recuperación hay que enmarcarlas dentro de una atención precoz; la evolución del estado tonicomotor general; la evolución de la sensomotricidad bucofaringoesofágica, y el interés del paciente por inhibirlo.

Las técnicas más frecuentemente empleadas son las activas: en ellas el paciente ha de colaborar de forma voluntaria para llegar a una deglución automática de la saliva. En algunos casos esta terapia funcional no es suficiente $\mathrm{y}$ hay autores que recurren a estimulaciones eléctricas, operaciones sobre las glándulas salivales y colocación de prótesis dentro de la boca, obteniendo — según su valoración- un alto porcentaje de éxito. Hemos de reconocer que, a pesar de lo prometedor de estos trabajos y de que realmente revisten un particular interés, su aplicación no es todavía suficiente como para generalizarla.

\section{LA RESPIRACIÓN}

En el mecanismo respiratorio normal, la respiración se adapta de forma automática a la longitud de la frase: a priori, y sin necesidad de pensarlo, ya conocemos el aire que va a ser necesario.

En la P.C., los reflejos tónicos y el tono muscular anormal dificultan la adaptación automática; ello plantea muchos problemas de respiración.

Como anomalía frecuente en la P.C. infantil, vemos espiraciones rápidas y superficiales; no saben inspirar profundamente. Muchas veces hacen una inspiración en extensión y luego tienen dificultades para realizar una espiración sostenida y profunda. Muchas veces encontramos un diafragma bloqueado por unos abdominales que no realizan su función. Por otro lado, a nivel de fonación vemos que, en ciertos intervalos, sólo hay emisión de ruido y no emisión vocal: el 
habla se produce sin aire, en muchos casos se puede constatar la disfonía.

En ocasiones, el no poder respirar por la nariz repercute en la alimentación, pues ya conocemos la coordinación que se establece entre la succión, la deglución y la respiración.

Los espásticos, por ejemplo y debido a los espasmos de los extensores del tronco, en ocasiones no podrán emitir la voz, aunque luego la producirán de golpe.

\section{M etodología del trabajo de la respiración y de la emisión vocal}

En el paralítico cerebral la respiración se trabaja de forma global, muchas veces ligada a la fonación. En la mayoría de los casos, si se intenta trabajar analíticamente la respiración, lo único que se consigue es su propio bloqueo o favorecer un patrón anormal de movimiento.

El objetivo es llegar a un trabajo automático y correcto de los músculos que intervienen en la respiración y en la emisión de voz.

Posición base: trabajo con el niño en decúbito supino; decúbito lateral izquierdo y derecho, y decúbito prono.

Colocamos las manos sobre el tórax y, en el momento en que empieza la espiración, la favorecemos con una vibración.

Para la fonación trabajamos la emisión sostenida del sonido. Ejemplo: o, o, o, o, o. Se pretende amplificar y tonificar el sonido.

El trabajo de emisión vocal es muy diferente según el tipo de lesión, pero en general se busca:

- la emisión mantenida de sonido;

- las variaciones de tono e intensidad;

- una emisión de sonido fluida y sin movimientos asociados;

- una tonificación general de los músculos que intervienen, a fin de conseguir una mayor capacidad de emisión vocal;

- que el paciente sepa sincronizar bien los diferentes movimientos y sepa relajar cada una de las partes del cuerpo implicadas en la emisión vocal cuando corresponda.

\section{Técnica del trabajo de respiración}

Objetivos: producirla y regularla automáticamente; que se adapte a la longitud de la frase, y que constituya una adaptación inconsciente.

Desarrollo de la terapia de respiración: tratar al niño de forma global; ir de la posición más fácil a las más difíciles.

\section{FACILITACIÓN DE LA RESPIRACIÓN DEL NIÑO (fig. 6):}

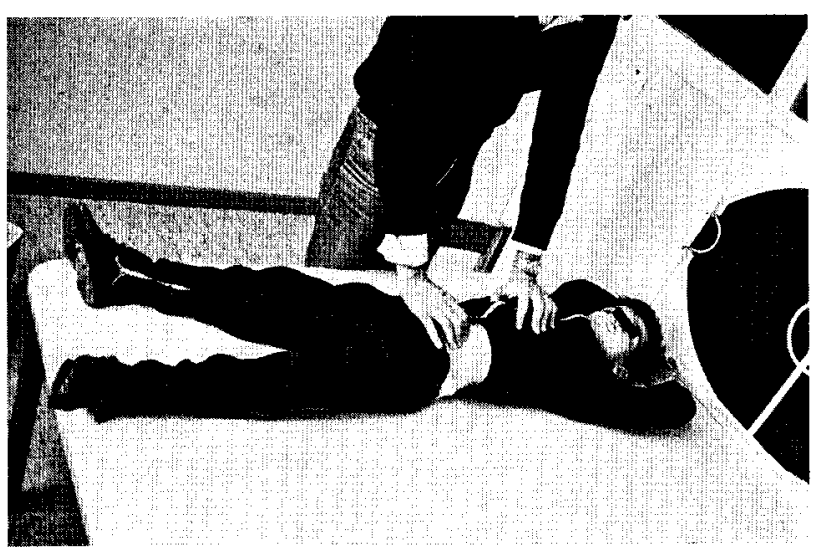

FIG. 6

Situar las dos manos sobre el tórax y notar su ritmo respiratorio.

En el momento de la espiración, ligera presión sobre el tórax.

Después de la espiración, mantener las manos sobre el tórax con presión.

En el inicio de la inspiración, suprimir la presión sin levantar las manos.

\section{TRATAMIENTO RESPIRATORIO Y DE LA FONACIÓN}

En la mayoría de casos, el tratamiento respiratorio irá acompañado del de la fonación. Si añadimos vibraciones en el momento de la espiración conseguiremos que el sonido salga más fácilmente.

Orden de los sonidos: O, U, E, I.

\section{Ejercicios}

Posición: niño en supino, piernas flexionadas, brazos a lo largo del cuerpo. 
Logopeda: delante del niño, manos sobre el tórax y el diafragma.

Objetivo: trabajar la inspiración nasal y espiración por la boca. En este caso, sólo se trabaja respiración.

Niño en decúbito lateral, primero de un lado y luego del otro (fig. 7):

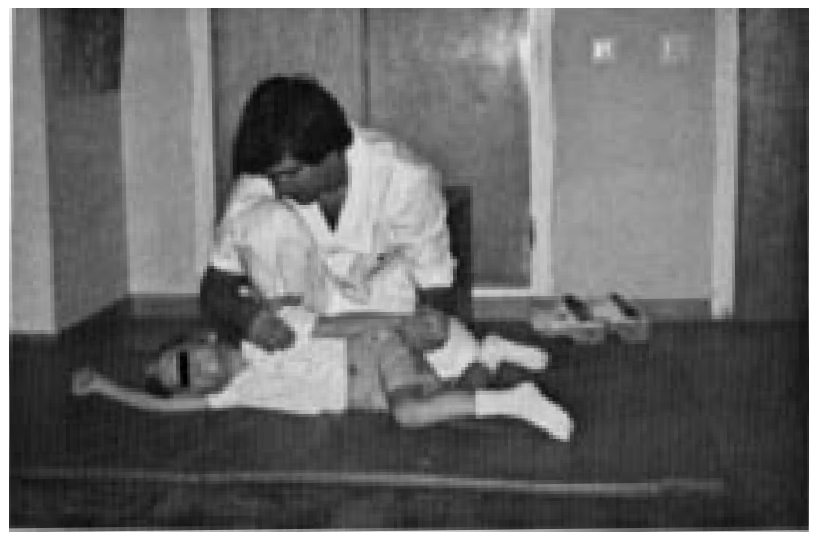

FIG. 7.

Niño en prono (fig. 8):

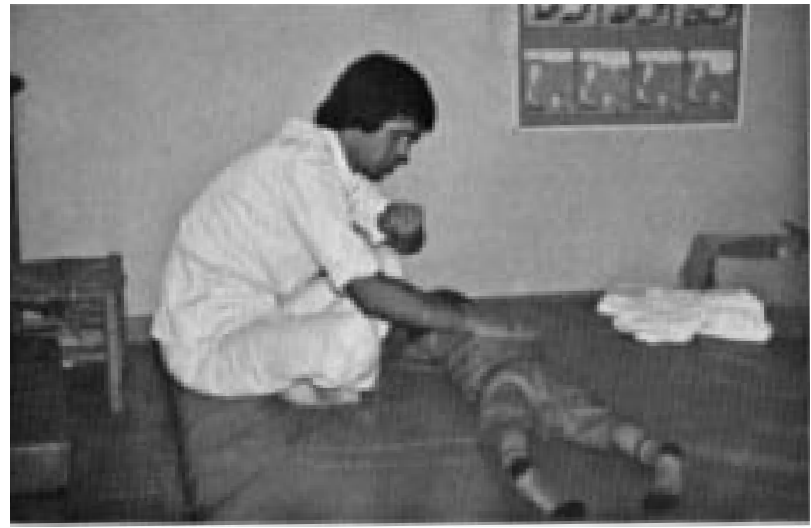

FIG. 8.

Niño sentado sobre la colchoneta o sobre una mesa. Logopeda: detrás del niño.

Llevar los brazos del niño hacia arriba, en rotación externa.

Tirar + vibración, a la vez que se hace notación de tronco.

Niño: sentado.
Logopeda: actúa sobre los hombros, aplicando una vibración y rotación. Puede hacerse también sobre la pelota (fig. 9).

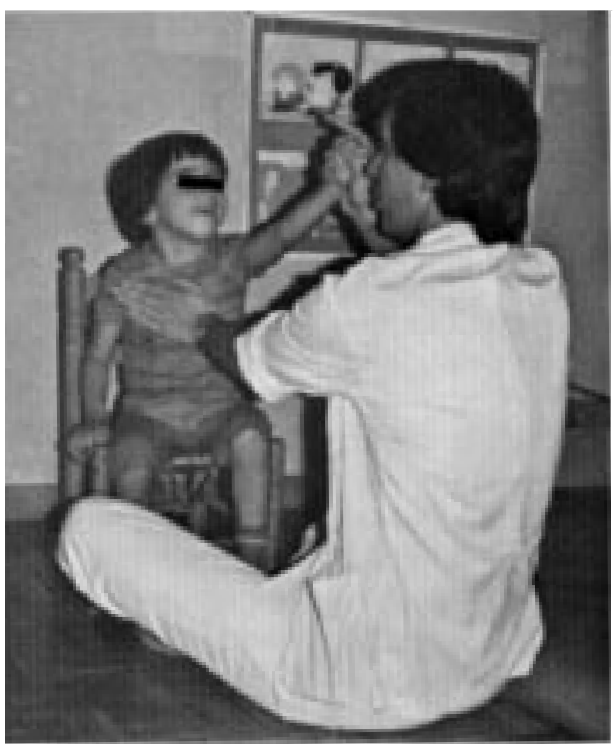

FIG. 9.

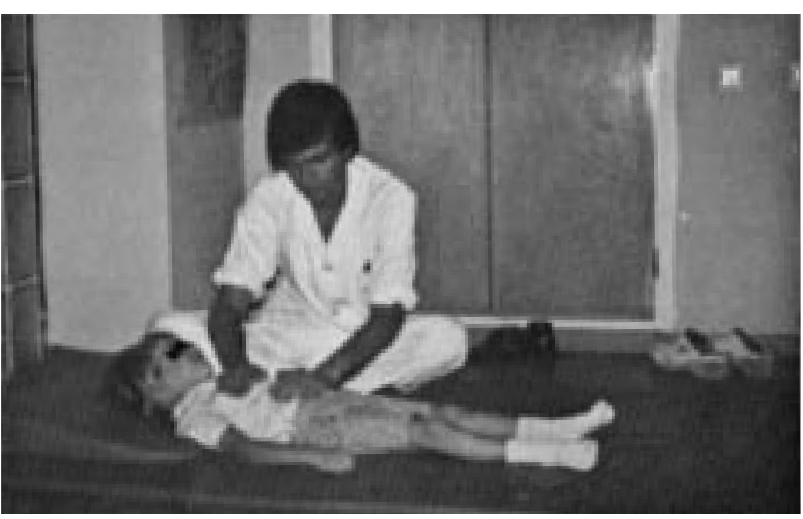

FIG.10.

\section{FONACIÓN}

\section{Posiciones}

- En supino con una cuña de fisioterapia debajo del tronco. 


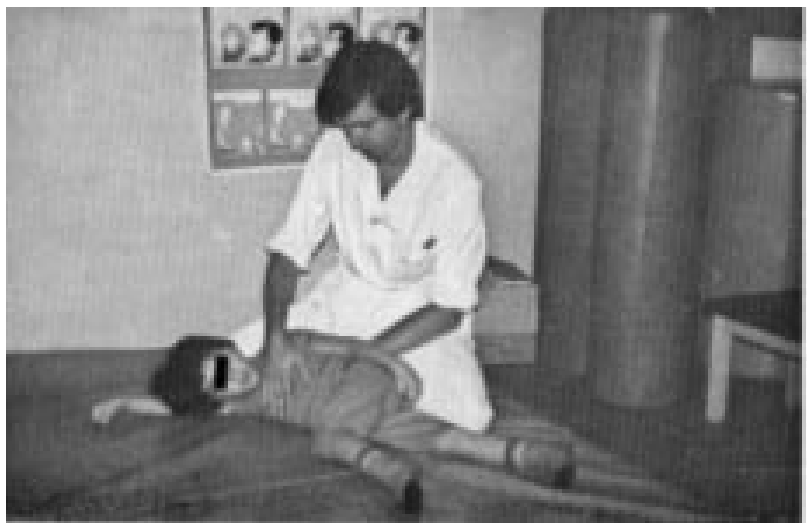

FIG. 11.

En esta posición, se trabajará la respiración y la emisión vocal sostenida (fig. 10).

- En caso de niños pequeños o muy afectados puede ser muy útil adoptar otra posición, con el niño sobre el terapeuta. Esta postura tiene grandes ventajas, pues nos permite controlar los movimientos del paciente y él puede ver con claridad nuestra boca.

- Favorecer la fonación en decúbito lateral derecho e izquierdo y también en prono (fig. 11).

- Más adelante, trabajo sentado, de pie, e incluso andando (figs. 12 y 13 ).

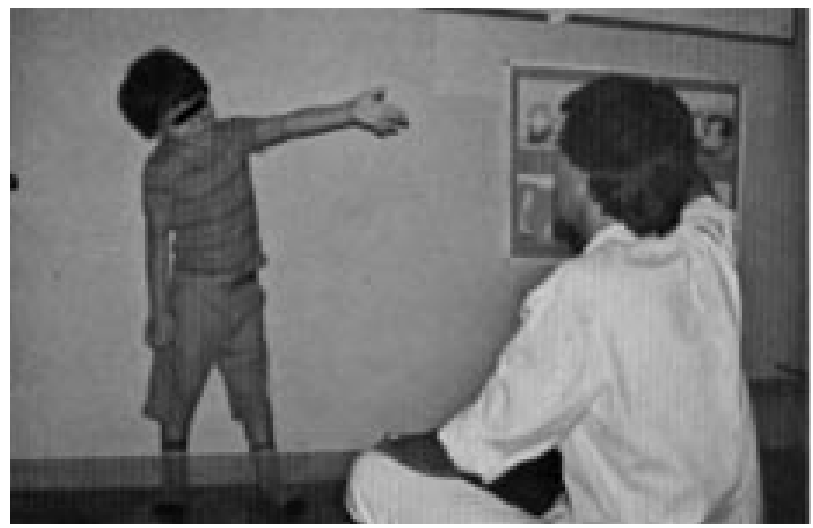

FIG. 12 .

\section{Emisión de sonido}

Procurar sobre todo que la ejecución del sonido sea flexible: que no fije la boca, que no haga un es- fuerzo exagerado a nivel de los repliegues vocales. No esperar a terminar la emisión; cuando se quede sin aire, interesa finalizar un poco antes.

No estar mucho tiempo con el mismo sonido, pues existe el peligro de fijación.

No tener prisa: precisamente una ejecución un poco lenta implica un mayor control motor. Por ejemplo, es más fácil llevar la lengua alternativamente de derecha a izquierda, según un movimiento regular, que llevarla despacio a la izquierda, volver al centro, parar durante cinco segundos y llevarla a la derecha.

Es más fácil emitir una $\mathrm{O}$ sostenida, que hacerlo y luego pasar a una E con la misma intensidad.

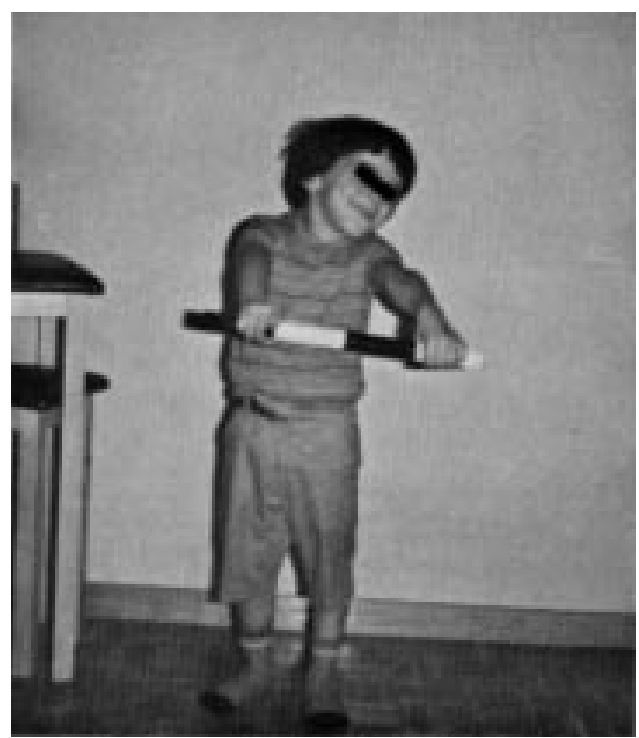

FIG. 13.

\section{Ejercicios orientativos}

Para que el paciente llegue a realizar los ejercicios que le pedimos, podemos reforzar su actividad con una serie de dibujos: éstos suelen manifestarse muy útiles para mantener el interés, sobre todo en casos de niños de menos de 6 años.

EMISIÓN SOSTENIDA DE SONIDO: O, U, I, E, A

- O: Viaje en coche a casa de los tíos (fig. 14). 


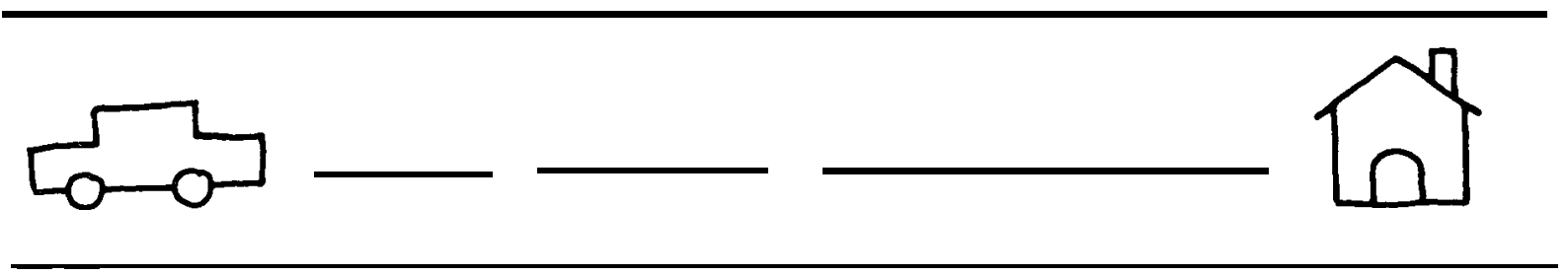

FIG. 14.

Marcar el trayecto con rotulador de diferentes colores. «Por la noche después de jugar con los primos, volvemos a casa» (marcarlo con otro rotulador).

- U: Viaje en tren (fig. 15).

«Vamos al pueblo de papá, donde nos esperan los abuelos.» Emplear igualmente los rotuladores.

- E: Viaje en barco (fig. 16).
«Has ido con la "ta-ta" a casa de los primos, que viven en Barcelona, en Palma... ¡Mira, ya te están esperando en el puerto!»

- Viaje en avión (fig. 17).

En los anteriores ejercicios se buscaba la emisión sostenida de un sonido. Ahora lo haremos cada vez con un mismo sonido, pero variando la intensidad.

Ejemplo: acabamos de llegar al aeropuerto y hay

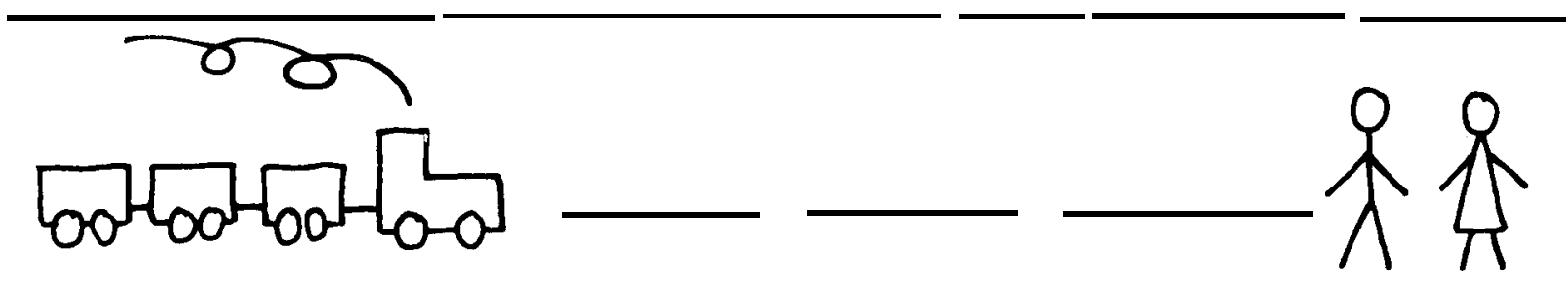

FIG. 15.

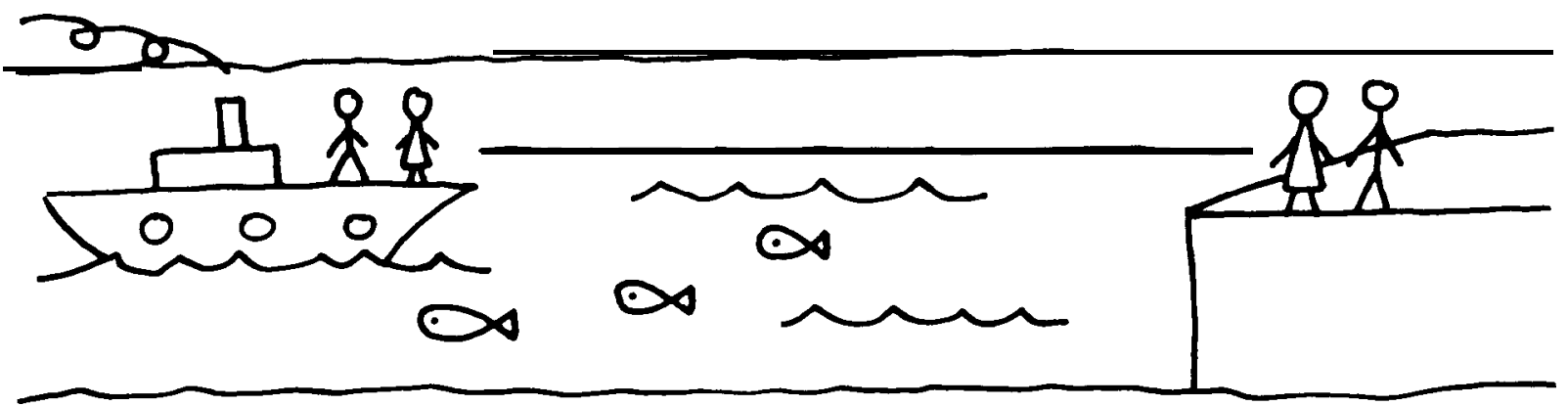

FIG. 16. 

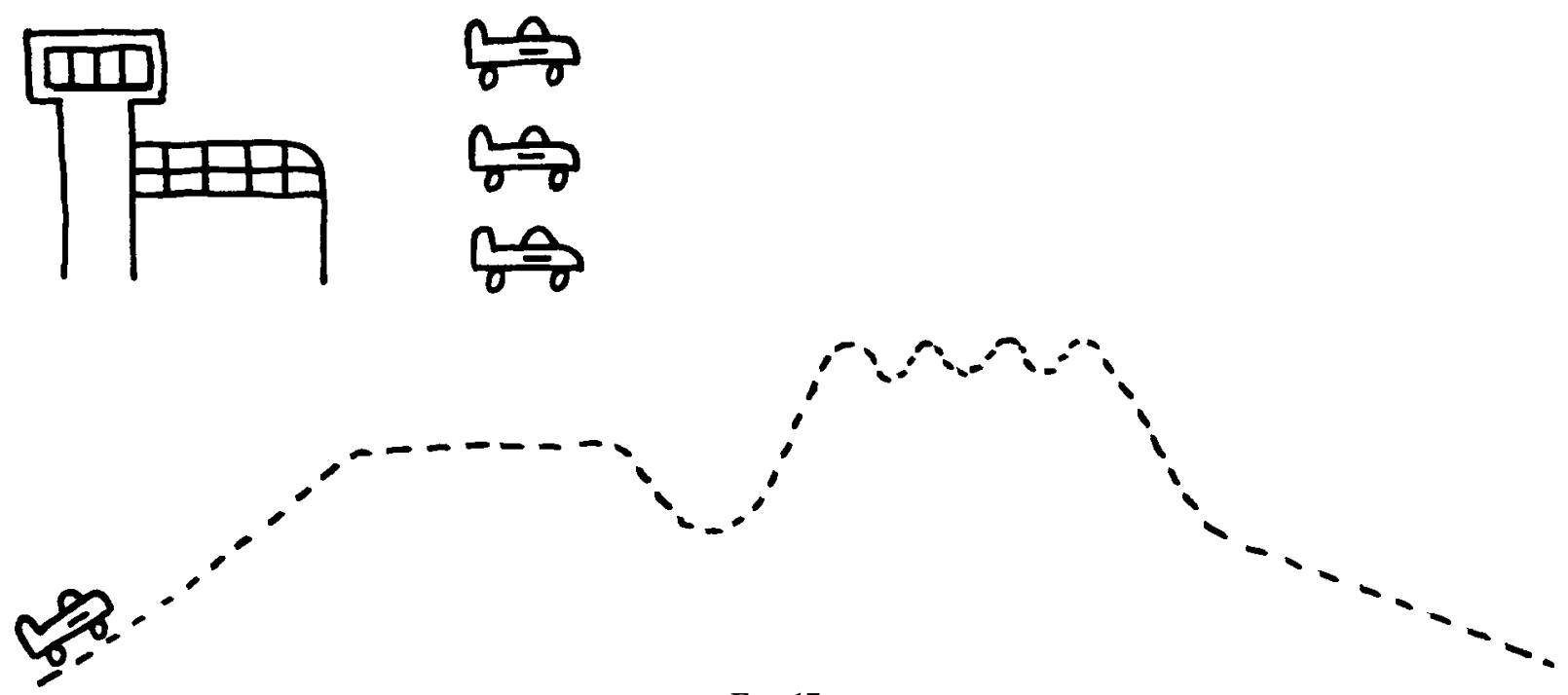

Fig. 17.

tres aviones: el 1 que es verde, el 2 que es rojo y el 3 que es azul (tener preparados los correspondientes rotuladores). Avión 1: sonido O. Avión 2: sonido E. Avión 3: sonido A.

- Excursión a la montaña. «Vas con el colegio y andando» (fig. 18).

En el ejercicio se pueden emplear varias secuencias rítmicas con sílabas sencillas y pequeñas variaciones de intensidad.
Ejemplo: po-po-po / ta-ta-ta / ba-ba-ba / ¡Ya hemos llegado!

- Excursión al parque: un sábado con papá (figura 19).

Se introducen algunas palabras de pocas sílabas muy utilizadas. Se busca automatizar la emisión y darle cierto ritmo.

Ejemplo: ¡vamos!-¡vamos! / ¡ya!-¡ya! / ¡adelante! / ¡ay! ¡ay! / ¡ay! / ¡ya!-¡mira! / ¡allá!/ ¡o, o, o, o!

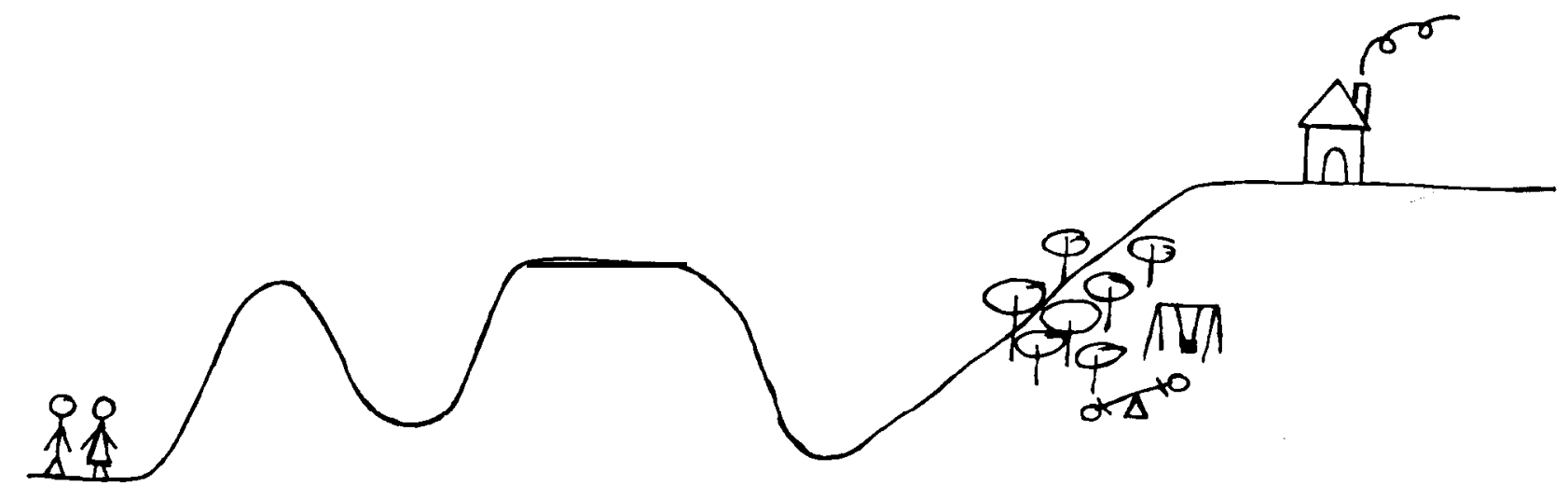

FIG. 18. 


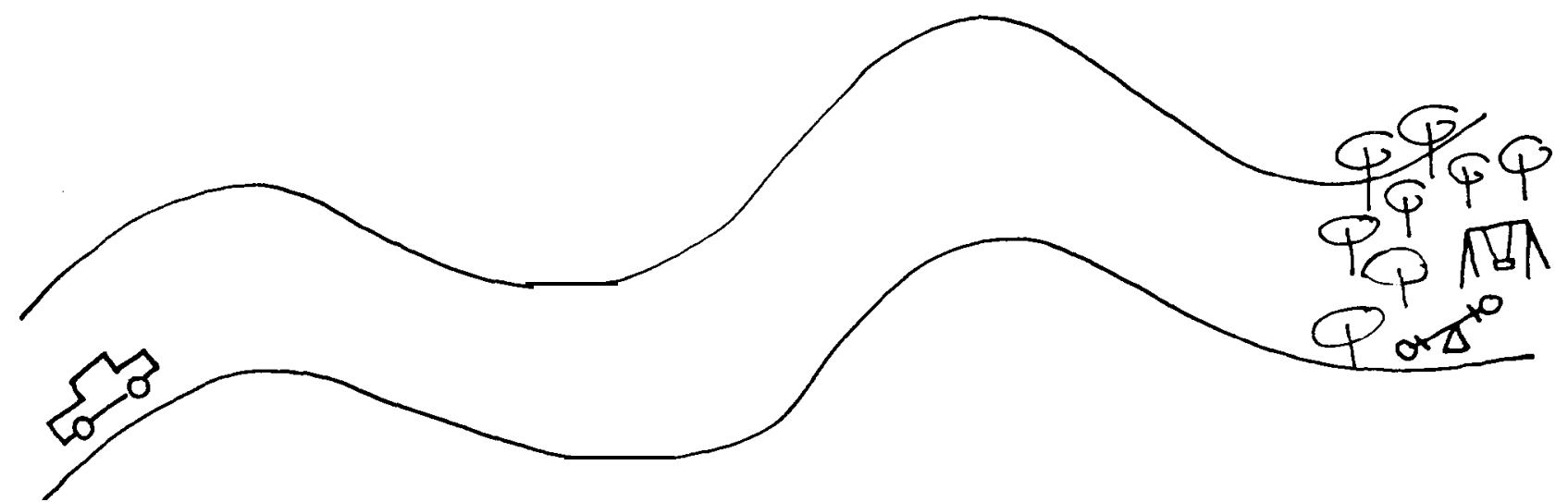

FIG. 19.

\section{Trabajo de la emisión vocal}

En el P.C. es fundamental, pues en la mayoría de los que tienen problemas de habla, ésta va unida a un patrón defectuoso de emisión vocal, y en muchos va acompañada de disfonía (véase Perelló, Viscasillas, Puyuelo, 1982).

Se trabaja en posición echado, sentado, de pie con y sin apoyo exterior y andando.

Según el momento del tratamiento nos entretendremos más o menos en cada una de las posiciones.

Tipos de voz: susurrada, normal, proyectada.

Variaciones de voz: tono, intensidad.

\section{DESARROLLO DE LOS EJERCICIOS}

Se empezará con las vocales, por ejemplo con la $\mathrm{O}$, haciendo ejercicios de voz «salmodiada», alargando en ocasiones el final si interesa. Luego con otras vocales.

Más adelante se hará el mismo ejercicio de voz salmodiada, pero con frases cortas y que tengan cierto interés para el paciente.

Ejemplo: «Voy a casa de mis primos.» «Jugaremos con el tren.»

- Por el contrario se pueden hacer ejercicios como «masticando las palabras».

Ejemplo: Hoy-e jugado-con-mis primos-y-sabes tenían-coches-de carreras.
- Es al rehabilitador a quien corresponde saber cuándo ha de emplear cada uno.

- Se empieza por ejercicios sencillos con palabras fáciles de realizar. Al principio, el paciente imita al reeducador pero poco a poco se debe intentar que los incorpore por sí mismo automáticamente a su habla. Los resultados de estos ejercicios, junto con los de dicción, son bastante positivos.

En este punto interesa naturalmente que las cualidades de emisión vocal del terapeuta sean buenas, pues el paciente va a imitar casi inmediatamente nuestro tono, nuestro ritmo...

\section{Ejercicios de dicción}

Su papel es muy importante en el tratamiento y se van empleando de forma progresiva en función de la evolución de la emisión vocal del paciente. Los métodos también son diferentes según su lesión.

En general a través de una lentificación del discurso y con unos patrones de articulación que se enseñan, el discurso del paciente mejora notablemente en inteligibilidad. Según el tipo de lesión se busca un mayor apoyo de los puntos de articulación o una mayor flexibilidad del gesto articulatorio, por medio de ejercicios de voz proyectada, salmodiada...

En las primeras fases del tratamiento, se practica sólo con el logopeda y en familia y más adelante en 
grupo, con otros niños y, como fase final, con un auditorio.

\section{M étodos de comunicación no verbal}

Cierto número de niños no podrán llegar a producir un mínimo de fonemas, ni mucho menos producir palabras de dos o tres sílabas. Sin dejar de trabajar las posibilidades de emisión vocal, el logopeda debe emplear los métodos de comunicación no verbal, y decidir en cada caso el más adecuado.

Algunos de los que se pueden emplear son:

- Pictogramas, símbolos que corresponden a los diferentes elementos del código lingüístico.

- El método Bliss, ya conocido, de símbolos más abstractos.

- Los silabarios, con las sílabas más utilizadas para que el paciente las pueda señalar para expresarse.

- Máquinas de escribir adaptadas, que distribuyen las casas especializadas.

- Sistemas electrónicos; por medio de ellos, hasta el paciente más afectado puede llegar a escribir, componer música, realizar ejercicios de orientación espacial todo ello con mandos teledirigidos y diferentes programas de ordenador.

\section{CONCLUSIÓN}

El tratamiento estará en función del tipo de lesión, del grado de afectación, del nivel psicoafectivo y del momento evolutivo. Por ello es muy diferente, a nivel motor, el tratamiento de una tetraparesia, una diplejía, o una hemiplejía; frente a una tetraparesia enfocaremos de forma diferente un caso con una afección motriz grave y otro con una afección motriz leve. Como dicen los autores anglosajones, el terapeuta ha de realizar en cada momento un «testing and treatment». El diagnóstico, en realidad, se renueva cada día. Un pequeño ejemplo lo puede constituir la «capacidad de emisión vocal», que en la persona «normal» es diferente según la fase del día; en el P.C. esto se multiplica.
En relación con el nivel psicoafectivo podemos encontrar casos en los que no sólo está alterado el aspecto de aprendizaje y «adquisiciones» sino el propio desarrollo afectivo, con diversos problemas según las edades: integración escolar, vida social, independencia personal, aspectos laborales...

Podríamos decir que durante los primeros años, sin prescindir de un enfoque global de la rehabilitación, se debe insistir más en las adquisiciones sensomotrices, lenguaje... para, de forma gradual, ir dando más importancia a los aspectos de integración social. Por ello, cuando el niño empieza a ir a la guardería intentamos reducir el número de sesiones especiales: logopedia, fisioterapia, terapia ocupacional, piscina, operaciones diversas...: realmente se podría decir que muchos tienen la agenda muy cargada, y ello en muchas ocasiones casi no les deje tiempo para jugar con niños de su edad. En ocasiones también podríamos hablar de un cierto síndrome de Supermán, pues a estos niños desde pequeños se les obliga a un gran sobreesfuerzo para ir superando sus dificultades. Nosotros hemos encontrado niños de 2 y 3 años cuyos juguetes, todos, eran de tipo «didáctico», para mejorar su destreza manual; y carecían de otros juguetes más sencillos o simplemente de objetos con los que inventar algo.

Más adelante la posibilidad de acceso a grupos de «Tiempo libre», de excursionismo... podrá suponer un importante estímulo. Hay que tener en cuenta aspectos como la independencia familiar y la integración social.

\section{RESUMEN}

La rehabilitación logopédica del paralítico cerebral, debido a lo heterogéneo de los casos que se presentan, requiere la aplicación de una gran diversidad de técnicas, y la habilidad del rehabilitador para escoger en cada momento la más adecuada. Por otro lado, es muy importante tener en cuenta siempre el objetivo último de la reeducación, las posibilidades del paciente y sus intereses. El fin de toda reeducación es que el paciente pueda tener experiencias y relaciones sociales normales. Debemos tener también 
en cuenta que los objetivos logrados durante la sesión individual deben generalizarse y éste no es un aspecto sencillo en la P.C. También debemos evitar una excesiva dependencia del paciente con respecto al rehabilitador, favorecida por el largo tiempo de reeducación. En ocasiones va bien el cambio de rehabilitador al cabo de unos años así como plantearse que la rehabilitación no puede durar toda la vida.

\section{BIBLIOGRAFÍA}

Bardier, J.: Le phoniatre et 1'I.M.O.C. En: Auzac: L'enfant infirme moteur d'origin cerebral. 2. ${ }^{\mathrm{a}}$ ed., Masson, París, 1980.

BARraquer Bordas, L. y WiEck, H. H.: Neurología en esquemas. Ed. Aesopus, Barcelona, 1975.

Billou, O. y TABARY, J. C.: Handicap et informatique. Tome I. Motricité Cérébrale. Masson, París, 1980.

Воватн, В.: Actividad postural refleja anormal causada por lesiones cerebrales. Ed. Médica Panamericana, Buenos Aires, 1973.

BoвAтH, B.: Hemiplejía: valoración y tratamiento. Ed. Médica Panamericana, Buenos Aires, 1972.

Воватн, В. у Воватн, К.: Desarrollo motor en distintos tipos de parálisis cerebral. Ed. Médica Panamericana, Buenos Aires, 1976.

Bobath, B. y Kong: Trastornos cerebromotores en el niño. Ed. Médica Panamericana, Buenos Aires, 1976.

Bosley, E. y Teaching, M. A.: «The Cerebral Palsied to Chew». The Cerebral Palsy Journal, 1966.

Bouton, C. P.: Le développement du langage. Aspects normaux et pathologiques (2. ${ }^{\mathrm{a}}$ ed.). Les Presses de l'Unesco, Masson, París, 1979.

BRody, G. S.: «Control of drooling by translocation of parotid duct and extirpation of mandibular gland». Develop. Med. Child. Neurol., 1977, 19, 514-517.

Bussière, B., LAJus, M. y Verniolle, B.: Bilan des troubles sensorieles et moteurs entraînant des difficultés de langage chez les I.M.C. "Motricité Cérébrale. Readaptation Neurologie du développement». 1980, 1-2, pp. 61-67.

CLíniCA Mayo: Lenguaje y habla motora. En: Examen Clínico Neurológico. 2. ${ }^{\mathrm{a}}$ ed., 1980. Prensa Médica Mexicana, México, 1980.

Colbeau, Ph. y Justin, Ph.: Troubles perceptifs visuels chez l'I.M.C. Méthode d'approche. «Motricité Cérébrale. Readaptation Neurologie du développement». 1-3, 128-144.

Comité Medical National de l'infirmité Motrice Cérébrale: Supplement au Bulletin des I.M.C., 1969, 55.

CORIAT, L. F.: Maduración psicomotriz en el primer año del niño. Ed. Hemisur S.R.L., Buenos Aires, 1974.

CoRiat, L. F.: Maduración neurológica del niño prematuro. En: Actualidades en Neuropediatría. Vol. I. Ed. Médica y Técnica, Barcelona, 1980.
CRIKMAY, M. C.: Logopedia y el enfoque Bobath en parálisis cerebral infantil. Ed. Médica Panamericana, Buenos Aires, 1977.

CHEVRIE, C.: Apprentissage gnoso-praxique et troubles moteurs en orthophonie. De I'organisation neurologique à la réalisation du langage. París, 1979. Ponencia del XX Congreso del C.D.I.

Chevrie, C.: Trastornos del lenguaje, la palabra y la voz. en el niño. 2. ${ }^{\text {a }}$ ed. En: LAunay y Borel-Maisonny, S. Editorial Toray-Masson, Barcelona, 1979.

Chevrie Muller, C.: «Paralysies et autres troubles de la motricité du voile du palais». Revue Française de prothèse Maxilo-Faciale. 1977, VI-I.

Chevrie Muller, C. y Hatt, A.: «Syndromes pseudo-bulbaires congénitaux et apraxies buceo-faciales congenitales. Interêt de I'enregistrement oscillographique de la parole». Arch. Franc. Ped., 1970, 27, 1019-1039.

Chevrie Muller, C. y Lebreton, M. T.: «Étude de la Réalisation des Consonnes au cours d'une épreuve de répétition de mots, sur des groupes d'enfants de 3 ans et 5 ans 1/2». Rev. de Laryngol., 1973, V. 94, 3-4.

Chevrie Muller, C.; Simon, A. M.; Florentin, F. y OliVIERS, M.: «Épreuves destinées a apprecier le niveau phonologique de l'enfant. Étalonnage pour les classes d'age 5 et 6 ans. Étude comparative des performances au cours des épreuves de répétition et de denomination spontanée». Revue de Neuropsychiatrie infantile, 1973, 21 (10-11), 593610.

Chevrie Muller, C., Simon, M. M. y Ballen, B.: «Exploration du langage chez l'enfant envisagé». Revue de Laryngologie, 1978, Vol. 99, $N .^{\text {os }} 7-8$.

CRYSTAL DAVID: Introduction to language. Pathologie. Arnold, Londres, 1980.

Gagnard, L. y Le Metayer, M.: Les techniques kinésithérapiques et la rééducation du langage parlé. En: Rééducation des infirmes moteurs cérébraux. Ed. Expansion Scientifique, París, 1979.

Goldstein KAREN JAFFE: Annual Abstracts of Speech, Voice, and Hearing. Little Brown and Company, Boston.

Grady Arnold, H. y Gross, Ch. W.: «Transtympanic Neurectomy: A Solution to Drooling Problems». Develop. Med. Child. Neurol., 1977, 9, 509-513.

Grenier, A., Vima, Ph. y Solomiac, J.: «Examen Neuromoteur complémentaire chez les nourrissons suspects d'infirmité motrice cérébrale». Cahier du C.D.I., 1976, 65.

Grenier, A.; Lesca, F.; Maurer, M. M. y Cazes de L.: Appareil Anti-Bavage. Cahiers du C.D.I., París, 1978.

HABERFEllner, H.: Autoterapia de trastornos sensomotores de la región oral. Posibilidades de mejoramiento simultáneo del control de posturas inadecuadas. XVI Congreso internacional de pediatría, Barcelona, 1980.

Haberfellner, H. y Rossiwall, B.: «Appliances For Treatment of Oral Sensori-Motor Disorders». American Journal of Physical Medicine, 1977. Vol. 56, N. ${ }^{\circ} 5$.

Haberfellner, H. y Rossiwall, B.: Treatment of Oral Sensoriomotor Disorders in Cerebral-palsied Children. 
Preliminary Report». Develop. Med. Child. Neurol., 1977, 19, 350-352.

Haberfellner, H. y HaffNer, B.: «Gestorte Mundfunkionen im Kindesalter». Pediat. Prax., 1979-1980, 22, 217-224.

HALLIDAY: Learning how to mean-Explorations in the development of langage. Edward Arnold, Londres, 1975.

HEAR y SAY: Toys for children with hearing speech and langage difficulties. Toy librairies association, London, 1978.

HerzKa, H. S.: Gesicht und Sprache des Sauglings. Ed. Schwabe, Basilea, 1979.

Illingwort, Ronald y Cynthia: Babies and Young Children, a Guide for Parents. 6. ${ }^{\mathrm{a}}$ ed. Churchill Livingstone, Edimburgo, 1977.

IngRam, T. T. S.: Clinical Significance of the infantile Feeding Reflexes». Develop. Medicine and and Child Neurology, 1962, 4.

Kong, E.: Les troubles de perception. En: Traitement et éducation de 18 enfant infirme moteur cérébral. Berna, 1979.

Levit, C.: Treatment of Cerebral Palsy and Motor Delay. Blackwell Scientific Publications.

Loebell, E.: Alteraciones de la audición y del lenguaje en niños con trastorno cerebromotor. En: BовATH y Kong: Trastornos cerebromotores en el niño. Ed. Médica Panamericana, Buenos Aires, 1976.

Martin, C. y Tabary, J. C.: La Psychometrie des I.M.C.

Mazet, Ph. y Houzel, D.: Psiquiatría del niño y del adolescente. Ed. Médica y Técnica, Barcelona, 1981.

Muller, H. A.: Terapia preparatoria del lenguaje. En: Bоватн у Kong: Trastornos cerebromotores en el niño. Ed. Médica Panamericana, Buenos Aires.

Muller, H. A.: Troubles de langage. En: Traitement et éducation de l'enfant infirme moteur cérébral. Berna, 1979.

Narcy, P. y cols.: Le Larynx de l'enfant. Société Française d'Oto-Rhino-Laryngologie et de Pathologie CervicoFaciale. Librerie Arnette, París, 1979.

PARDo, B.: De la sensorio-motricité a l'education thérapeutique du geste. Cahiers du C.D.I., París, 1979.

Perelló, J., Ponces, J. y Tresserra, L.: Enfermedades del sistema nervioso central, vol. I. Parálisis cerebral infantil. Ed. Científico-Médica, Barcelona, 1973.

Pialoux, P. y cols.: Manual de Logopedia. Ed. Toray-Masson, Barcelona, 1978.

Puyuelo, M.: El logopeda en el equipo de parálisis cerebral infantil. En: Publicación del 1. ${ }^{\text {er }}$ Symposium Nacional de Enfermería Pediátrica. División de Enfermería, Hospital San Juan de Dios, Barcelona, 1980.

Rizo, M.: Concepto de parálisis cerebral o trastornos cere- bromotores. En: Publicación del 1. ${ }^{\text {er }}$ Symposium Nacional de Enfermería Pediátrica. División de Enfermería, Hospital San Juan de Dios, Barcelona, 1980.

Rondal, J. A.: Lenguaje y Educación. Ed. Médica y Técnica, Barcelona, 1980.

Rondal, J.: La evolución del lenguaje. Ed. Médica y Técnica, Barcelona, 1982.

Rondal, J. A.: Lenguaje y Educación. Ed. Médica y Técnica. Barcelona, 1975.

Rossiwall, B., Haberfellber, H., Oberweger, H., ObenDORF, H.: Apparate zur Behandlung oraler Dysfunktion. 672, dental-labor, XXIV Jahrgang, Helt 5176.

Routledge, Linda: El niño con deficiencias físicas. Orientaciones para su tratamiento. Ed. Médica y Técnica, Barcelona, 1980.

SCHNEIDER, D.: Troubles de la respiration chez l'I.M.C. au cours de la parole. Cahiers du Cercle de Documentation et d'Information, núm. 57. París, 1973.

SCHNEIDER, D.: Troubles de la respiration chez l'I.M.C. au cours de la parole. «Cahier du Cercle de Documentation et d'Information pour la rééducation des infirmes moteurs cérébraux», 1973, 57, pp. 28-46.

SEGOVIA, M. L.: Interrelaciones entre la odontoestomatología y la fonoaudiología. La deglución atípica. Ed. Médica Panamericana, Buenos Aires, 1977.

Société Belge d'Oto-Rhino-Laryngologie et de Chirurgie cervico-faciale: «Classification décimale universelle-ORL». Acta Oto-Rhino-Laryngologica, Bélgica, 1981.

Tardieu y Alvergue, R.: Evaluación del lenguaje. En: Invalidez motriz cerebral (Parálisis cerebral con conservación de la inteligencia). Ed. Talleres del Instituto Salvadoreño del Seguro Social.

Tardieu Guy: Annexe III Feuille E.T.L. Clinique. En: Le dossier clinique de l'I.M.C. «Cahier du Cercle de Documentation et d'Information pour la rééducation des infirmes moteurs cérébraux». 1976, 39.

UNESCO: L'enfant et son développement de la naissance à six ans (mieux le connaitre pour mieux l'aider). Ed. Ateliers de l'Unesco, París, 1976.

VAN RIPER: La inteligencia de los paralíticos cerebrales. En: CRICKMAY: Logopedia y el enfoque Bobath en parálisis cerebral. Ed. Médica Panamericana, Buenos Aires, 1974.

Varios: Un buen principio prepara un buen futuro. Vuestro hijo. Milupa, S. A., Madrid, 1979.

Varios: Psychobiologie de l'enfant en développement. Congrès international de psychologie de l'enfant. Documents, París, 1979.

Recibido: enero 1983. 